\title{
Vibrations of the "beetle" scanning probe microscope: Identification of a new mode, generalized analysis, and characterization methodology
}

\author{
Matthew J. Brukman and Robert W. Carpick \\ Department of Engineering Physics, University of Wisconsin, Madison, Wisconsin 53706
}

(Received 13 December 2005; accepted 5 February 2006; published online 31 March 2006)

\begin{abstract}
The prediction and understanding of structural resonances are required to optimize scanning probe microscope (SPM) design. Here, Euler beam theory is applied to the beetle-style SPM to derive analytic functions for the natural frequencies of three significant modes of vibration as a general function of the microscope shape, materials, and dimensions. In the first mode, the three piezoelectric legs vibrate transversely and the scanhead moves from side to side. In the second, the legs bend circumferentially and the scanhead rotates about its center. These modes have been identified previously, but here the mechanics analysis is presented in an improved form where the inertia of the piezo legs is considered, constraints on the shape of the central supporting disk are lifted, and appropriate boundary conditions are defined and enforced. In addition, we discuss a third mode that has not been previously identified. In this lowest frequency mode, two legs pivot about the stationary third leg. The predictions are tested against experimental data obtained from an atomic force microscope (AFM) built in our laboratory. We show that the mode frequencies can be determined easily using in situ motion of the AFM cantilever itself. Predicted frequencies are in good agreement with experimental results, although unpredicted modes are also observed. The simple closed-form solutions allow the designer to make quantitative comparisons when choosing the materials and dimensions used in the SPM design. Two new design criteria emerge from the analysis for optimizing the resonant response of the "beetle" scanhead: (1) the wall thickness $\tau$ of the piezo legs should be minimized, with their mean diameters increasing as $\tau^{-1}$ and (2) the distance between the legs and the center of the scanhead should be adjusted to optimize the rotation mode eigenfrequency. (c) 2006 American Institute of Physics. [DOI: 10.1063/1.2183266]
\end{abstract}

\section{INTRODUCTION}

The beetle-style scanning probe microscope ${ }^{1}(\mathrm{SPM})$ is a popular design that is used for atomic force microscopy (AFM) and scanning tunneling microscopy in both custombuilt and commercial systems. ${ }^{2}$ The advantages of this design include the relatively compact size and thus small mechanical loop, intrinsic symmetry that reduces effects of thermal drift, and the convenient incorporation of reliable fine and coarse tip approaches and microscope positioning.

In this configuration, the probe equipment is attached to a small thin disk, to which three piezoelectric hollow tube $\mathrm{legs}^{3}$ are symmetrically connected, usually via soldering. The legs in turn are each attached to spheres (usually glass balls) that rest on the sample holder, which is typically a helical ramp. ${ }^{4}$ The piezo legs are metal-plated to form four orthogonal outer electrodes and a common interior electrode. Voltages applied to these five electrodes induce transverse bending and axial compression or elongation via the inverse piezoelectric effect, endowing the SPM head with $x-y-z$ position control to raster scan the sample and navigate its topography. In this "scanning" motion, the balls remain fixed on the ramp. Alternatively, the entire head can be "walked" by inertial motion, ${ }^{5}$ whereby fast voltage drops cause the balls to slide with respect to the ramp, enabling. This enables coarse the positioning of the head for acquiring data over a range of locations on the sample. As well, by walking the scanhead clockwise or counterclockwise on a helical ramp, the SPM probe tip is brought gradually and safely into or out of contact with the sample. A schematic of our beetle AFM is shown in Fig. 1 and is based on the design by Dai et al. ${ }^{6}$ The walker uses lead zirconate titanate (PZT) actuators for the legs (PZT 5A, Staveley Sensors, East Hartford, CT).

Since SPMs are used to image samples with subnanometer spatial resolution, even very small amounts of mechanical noise may severely impair data collection. ${ }^{7}$ To attenuate ambient mechanical noise, SPMs are typically placed in basements, on vibration isolation tables, in soundproof chambers, and on suspension systems. Even when such precautions are taken, some vibrations are passed from the environment to the SPM, and so modeling its dynamic mechanical response is important. In the most elementary approach, the microscope may be modeled as driven oscillator with natural frequency $\omega_{0},{ }^{8}$ which in practical terms represents the lowest resonance frequency of the mechanical loop from the sample, through the apparatus, and back to the tip. At resonance, the tip and sample motions are out of phase, inducing positional error and corresponding noise in the measurement. The transfer function governing this interaction provides the amplitude ratio of SPM vibration to noise input from the environment and, in the case of a one-stage vibration isolation system, is proportional to $\left(\omega-\omega_{0}\right)^{-2}$ for $\omega>\omega_{0}$. To minimize mechanical noise, SPMs should be constructed with natural frequencies as high as possible. Of 


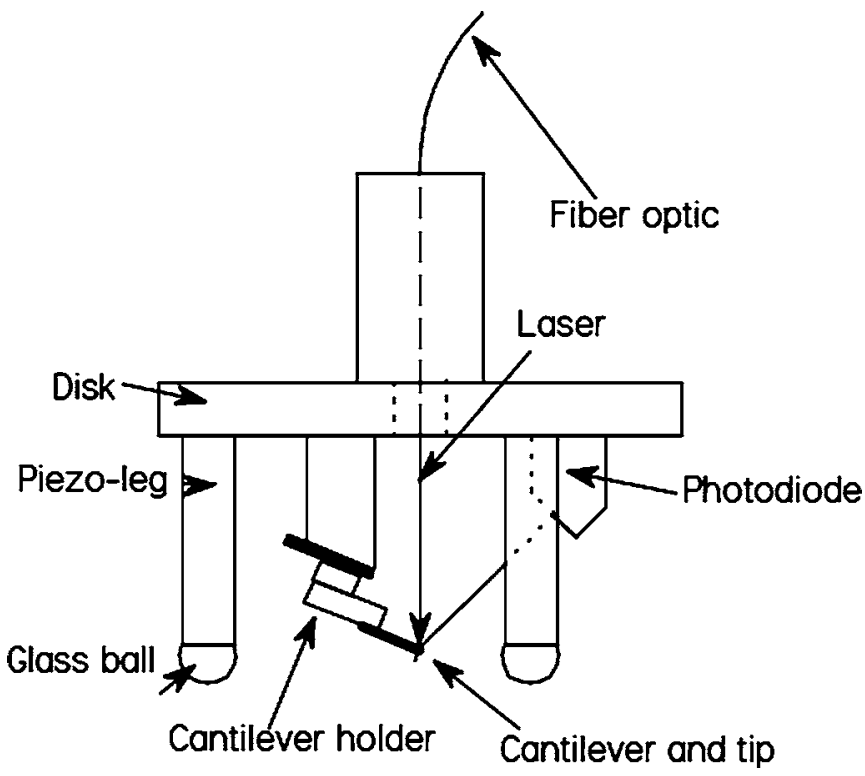

FIG. 1. Schematic of the beetle-style AFM scanhead. The photodiode lies between two piezo legs, with the rear leg hidden.

course, SPMs have many degrees of freedom and accordingly have many natural modes of vibration, each with its own frequency. The lowest natural frequency will have the greatest transfer function at a given input frequency and the designer's attention should be primarily focused on the one or two lowest frequency modes so that their frequencies may be optimized by making them as high as possible.

\section{ANALYSIS}

The goal of the analysis is to predict the resonance frequencies as a function of the dimensions and material properties of the head. We first calculate the bending mode shapes of the legs using Euler beam theory, and then find their kinetic and bending strain energies, which depend on those mode shapes. After the kinetic energy of the disk and probe equipment (i.e., lens, cantilever, and photodiode in the case of AFM) is added to the model, Rayleigh's quotient is used to predict the resonant frequencies of the vibrations. In the beetle design, the probe equipment is attached to a disk, to which the legs are soldered at one end. The other ends of the legs contact the ramp via glass or sapphire balls. In this configuration, the legs may be idealized as beams built-in to rollers at the disk end and pin-supported at the ball end. ${ }^{9}$ Considering the coordinate $z$ to run along the leg's axis from the disk $(z=0)$ to the ball $(z=L)$, as shown in Fig. 2, the boundary conditions for displacement $u(z)$ are thus (with subscripts indicating differentiation in $z$ )

At the connection to the balls: $u(L)=0$,

$$
u_{z z}(L)=0 \text {, }
$$

At the connection to the disk: $u_{z}(0)=0$,

$$
u_{z z z}(0)=0 \text {. }
$$

Equations (1a)-(1c) reflect the following mechanical constraints on the piezo legs, respectively: (a) they are

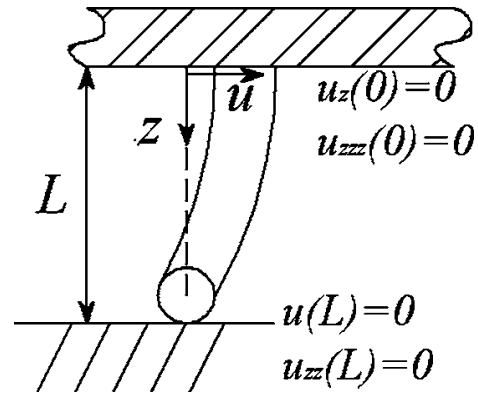

FIG. 2. Coordinate system and boundary conditions of piezo leg bending.

pinned at the stage $(z=L)$ and may not deflect transversely; (b) they are free to bend at the stage [the slope $u_{z}(0)$ can be nonzero] thus they do not support a bending moment at $z$ $=L$ (from beam mechanics, the second derivative of the transverse deflection is proportional to the bending moment); and (c) the legs are built-in to the disk and cannot bend at that point, thus there is no slope at $z=0$. Note that it is not required that $u(0)=0$, since the transverse deflection of the head is allowed.

Condition (1d) merits a separate discussion on the role of the scanhead mass. Here we postulate that the disk is free to displace laterally and thus cannot support a shear force at $z=0$ (from beam mechanics, the third derivative of the transverse deflection is proportional to the transverse shear force $^{9}$ ). Strictly speaking this assumption is not correct, as the shear force at the disk end is equal to the product of the mass and acceleration of the disk. The actual motion of a leg is a superposition of multiple normal modes and the addition of end masses to the beam shifts the eigenfrequencies and favor higher order modes of vibration. Because these modes are of greater frequency and reduced end displacement than the first mode, ${ }^{10}$ they are not relevant to the present analysis, which focuses on the more important low- $f$ modes. Furthermore, the homogeneous boundary condition (1d) allows a closed-form solution, whereas adding the end mass requires the numerical solution of a transcendental equation. We have verified that the mode shapes resulting from the two choices of boundary conditions are nearly identical and that the resulting eigenfrequencies agree within less than $1 \%$.

According to the Euler beam theory, ${ }^{11}$ the harmonic deflection of the leg is of the form $\nu(z, t)=A \sin (\omega t) u(z)$, where $u(z)$ is a solution of a differential equation,

$$
\frac{d^{2}}{d z^{2}}\left(E I \frac{d^{2} u}{d z^{2}}\right)-\rho \omega^{2} u=0 .
$$

Here, $\omega, E, \rho$, and $I$ represent the angular vibration frequency, Young's modulus of the leg material, density of the leg material, and cross sectional moment of inertia for the bending of the leg. The product EI is commonly referred to as the flexural rigidity. Equation (2) has a general solution $u(z)=A \cos (\lambda z)+B \cosh (\lambda z)+C \sin (\lambda z)+D \sinh (\lambda z) . \quad$ The boundary conditions (1) reduce this to the specific solution $u(z)=A \cos (\pi z / 2 L)$. The harmonic time dependence may be added to form $\nu(z, t)=A \sin (\omega t) \cos (\pi z / 2 L)$.

To carry the analysis further, we must identify the geometry of the natural modes of vibration of the head. We have identified three such modes (Fig. 3). Two of these, rotation 

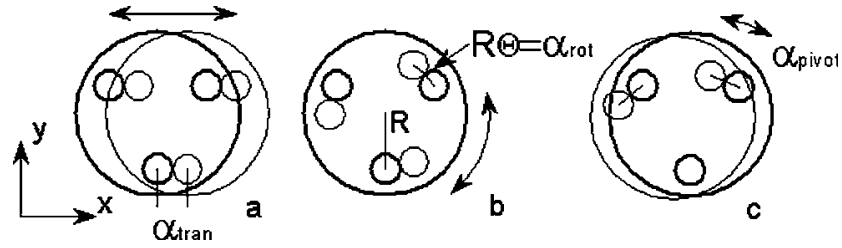

FIG. 3. Top views of the translational (a), rotational (b), and pivot (c) vibration modes of the scanhead.

about the central axis and lateral translation, have been described before. We also demonstrate the existence of a third mode that had previously been ignored, which we refer to as pivoting. The amplitudes $u(0)=A$ of the three low frequency vibration modes shall now be distinguished as $\alpha_{\text {tran }}, \alpha_{\text {rot }}$, and $\alpha_{\text {pivot }}$ (Fig. 3). In the translational case, all three legs deflect the same amount $\alpha_{\text {tran }}$ and in the same direction, translating the head laterally. This translation is free to occur in any lateral direction and could be considered as two degenerate eigenmodes for any two orthogonal axes in the plane of the disk. For rotation, the three legs cause the disk to rotate by deflecting circumferentially with respect to the disk by $\alpha_{\text {rot }}$ $=R \Theta$, where $R$ is the radial distance from the legs to the center of the disk (which we distinguish from the radius of the disk itself) and $\Theta$ being the angle of rotation. The third mode postulated, in which two legs bend about a stationary third leg, is a special case of the rotation mode, and will be discussed separately. With $A_{x s}$ the cross sectional area of a leg, the reference kinetic energy of the walker may be determined for translation and rotation,

$$
\begin{aligned}
& T_{\text {tran }}=3 \int \rho A_{x s}[u(z)]^{2} d z+M \alpha^{2}, \\
& T_{\text {rot }}=3 \int \rho A_{x s}[u(z)]^{2} d z+J \dot{\Theta}^{2},
\end{aligned}
$$

where $M$ is the true mass of the disk and probe equipment, and $J$ is the mass polar moment of inertia of the disk and probe equipment about the disk's central axis, which is assumed to be axially symmetric. The reference potential (strain) energy of an individual leg's bending is

$$
V=\int E I\left[\frac{\partial^{2}}{\partial z^{2}} u(z)\right]^{2} d z
$$

For a thin walled tube of inner radius $r$ and thickness $\tau$, $I$ may be approximated as $\pi r^{3} \tau$. Last, Rayleigh's quotient is applied to determine the frequencies, ${ }^{10}$

$$
\begin{aligned}
& \omega_{\text {tran }}^{2}=\frac{V}{T_{\text {trans }}}=\frac{3 E I \pi^{4}}{32 L^{3}\left(M_{\text {disk }}+\frac{3}{2} M_{\text {leg }}\right)}, \\
& \omega_{\text {rot }}^{2}=\frac{V}{T_{\text {rot }}}=\frac{3 E I \pi^{4}}{32 L^{3}\left(J / R^{2}+\frac{3}{2} M_{\text {leg }}\right)},
\end{aligned}
$$

or in units of Hertz,

$$
f_{\text {tran }}=\sqrt{\frac{3 E I \pi^{2}}{128 L^{3}}} \div \sqrt{M_{\text {disk }}+\frac{3}{2} M_{\text {leg }}},
$$

$$
f_{\text {rot }}=\sqrt{\frac{3 E I \pi^{2}}{128 L^{3}}} \div \sqrt{\frac{J}{R^{2}}+\frac{3}{2} M_{\mathrm{leg}}},
$$

where $M_{\operatorname{leg}}=A_{x s} L \rho$.

Applied to the dimensions of our AFM scanhead, (8) and (9) predict the translation mode resonance at $1450 \mathrm{~Hz}$ and the rotation mode resonance at $1920 \mathrm{~Hz}$. Comparisons to experimental measurements are discussed in the next section. The mechanics of the third, or pivot, mode are very similar to the rotation mode, with two distinctions. First, the strain and kinetic energies of bending are each reduced by one third, as only two legs bend. Second, the axis of rotation is shifted from the center of the scanhead to the axis of one of the piezo legs. This mode $f_{\text {pivot }}$ is therefore predicted by modifying Eq. (9), replacing both 3's with 2's to account for the number of bending legs and replacing the $J$ with $J+M R^{2}$ according to the parallel axis theorem. Thus,

$$
f_{\text {pivot }}=\sqrt{\frac{E I \pi^{2}}{64 L^{3}}} \div \sqrt{\frac{J+M_{\text {disk }} R^{2}}{R^{2}}+M_{\text {leg }}} \text {, }
$$

which predicts a pivot excitation at $970 \mathrm{~Hz}$. We anticipate that as written, Eq. (10) is a slight underestimation of the actual $f_{\text {pivot }}$, as the stationary leg has some torsional stiffness, depending on the strength of the contact with the ramp, which is not considered in the present analysis.

The equations for translation and rotation mode resonances are similar in form to the result of Behler et al., ${ }^{12}$ but there are four meaningful distinctions in this analysis. First, a dynamic analysis is present here, meaning that the masses of the legs are considered. For the head we have constructed, the piezos add approximately $10 \%$ to the masses of the second radicals in each equation, resulting in frequency corrections of $3 \%$ for $f_{\text {tran }}$ and $5 \%$ for $f_{\text {rot }}$. However, the correction may not be so modest for other designs, In fact, one way to improve SPM designs is to reduce the mass of the disk and probe equipment through the use of low-density materials or the efficient distribution of the mass, thereby increasing the relative importance of the legs' mass and rendering its inclusion in these formulas more critical. For example, if the disk and probe elements are made from Be (which has an excellent modulus to density ratio) and the disk dimensions reduced to take advantage of Be's greater modulus to prevent vibrational modes of the disk itself from changing, then the mass correction would be $18 \%$ and would be even larger with the further optimization of the disk's vibration modes (see Appendix).

Second, the boundary conditions (1) enforced here result in different "spring constants" for the individual piezo tubes. Behler treated the legs as fixed-free cantilever beams, meaning that two of the four boundary conditions were incorrect. It is fortuitous that this choice results in an error of merely $1.5 \%$ [the difference between $3 E I / L^{3}$ and $\left.\pi^{4} E I /\left(32 L^{3}\right)\right]{ }^{13}$ As noted previously, the boundary condition (1a) assumed here is itself an approximation that introduces a small error, because the coupling between the stage and each ball on the end of the piezo legs is not perfectly rigid. Rather, the contact region forms a lateral spring of finite stiffness determined by surface roughness and composition and ball radius. ${ }^{14}$ 


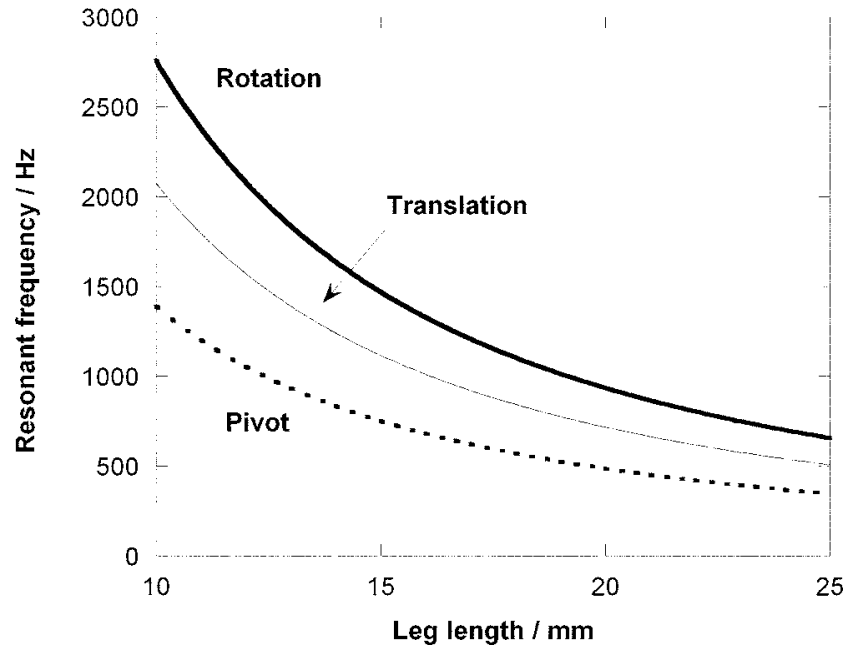

FIG. 4. The natural frequency of each mode will decrease as piezo leg length $L^{-1.5}$ (values are for the scanhead described in the text and Ref. 19).

Also, Behler et al. ${ }^{12}$ and later MacLeod et al. ${ }^{15}$ and Miwa et al. ${ }^{16}$ assumed the simple geometry of a cylindrical disk with three legs attached exactly at its outer radius, resulting in a constant difference by a factor of $\sqrt{2}$ between $f_{\text {tran }}$ and $f_{\text {rot }}$. Equations (8) and (9) show that this is not the case, as $f_{\text {rot }}$ has an explicit $R$ dependence, while $f_{\text {tran }}$ does not. In practice, the probe equipment significantly departs from this idealized form, especially for an AFM, which requires an off-axis cantilever holder and photodetector. As computer-aided design packages can be used to calculate $M$ and $J$ of the disk/probe assembly, the simplifying assumption is no longer needed from a computational standpoint. Therefore, leaving $J$ as a parameter in the present analysis allows designers to take advantage of this and perform more accurate calculations.

Last and most critical, there has been no previous discussion of the pivot mode, which has a lower frequency than either translation or rotation modes and is therefore more likely to be excited by ambient mechanical noise. The rotation and translation modes may be viewed as the limiting cases of the pivot mode for small or large $R$, respectively, although those two modes will always have greater eigenfrequencies than the pivot mode by at least a factor of $\sqrt{3 / 2}$, so the introduction of the pivot mode represents a base line correction of $22 \%$.

These explicit formulas for three low frequency, highamplitude modes will aid the designer of a beetle-style SPM. In particular, the following conclusions may be drawn:

(1) When deciding on the length of the piezo leg, the designer must weigh vibrational response against piezo range (displacement per applied volt). The piezo range increases as $L^{2}$, but the resonant frequencies fall as approximately $L^{-1.5}$. Figure 4 shows the predicted variation of natural frequency with piezo leg length for our AFM head.

(2) It is advantageous to increase the flexural rigidity $E I$ of the legs; $E$ is a given property of the piezoelectric, but there are two degrees of freedom in $I$. As stated above, for a thin walled tube of inner radius $r$ and thickness $\tau$,

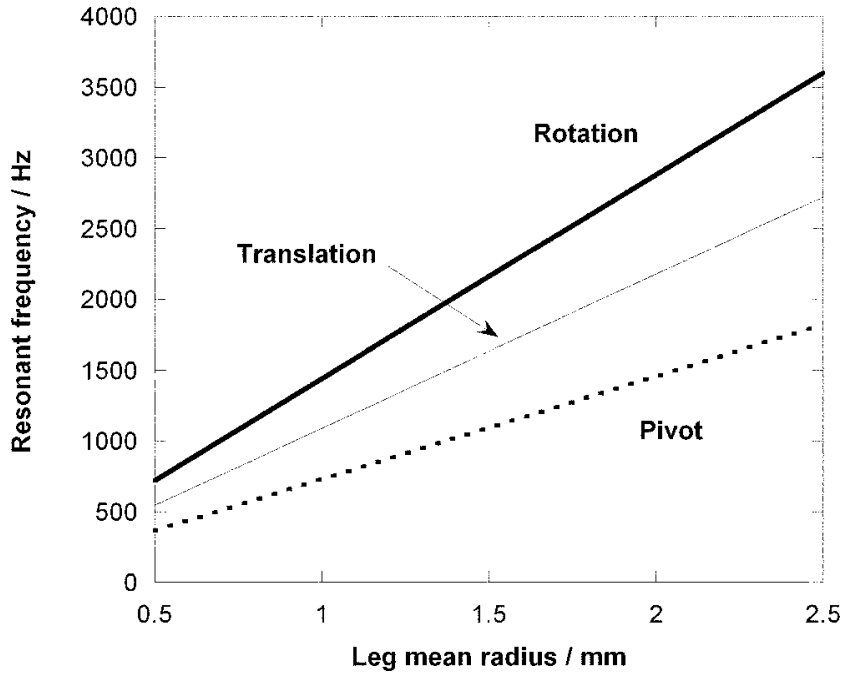

FIG. 5. The natural frequency in each mode will increase if the piezo legs' cross section areas are kept constant while their radii increase and thicknesses decrease (values are for the scanhead described in the text and Ref. 19).

$I$ may be approximated as $\pi r^{3} \tau$, so both resonance frequencies should increase, to first order, as $r^{1.5}$. However, the leg mass $M_{\text {leg }}$ is proportional to the leg's cross sectional area, which also increases with $r$, equaling $2 \pi r \tau L \rho$ for a thin tube. If the product $r \tau$ is kept constant while $r$ is increased, then $f$ will increase linearly with tube radius (Fig. 5). Because the lateral piezo sensitivity varies as $1 / r \tau$, following this rule and making the tubes thinner increases the stability with no trade-off in lateral scan range, while vertical scan range will increase as $1 / \tau$. However, electric fields within the piezos increase as the walls become thinner, so depoling will then become the limiting factor. Typical PZT piezos have dc depoling fields of at least $200 \mathrm{~V} / \mathrm{mm}$, so allowable tube thicknesses may be $1.2 \mathrm{~mm}$ or less for $\pm 240 \mathrm{~V}$ power supplies. Thin, wide-diameter piezo tubes will thus provide better mechanical performance. One caveat is that actual piezo tubes may not be thin enough for these approximations to be completely valid. If the tubes are of modest wall thickness, then $f(r)$ will level off at small radii.

(3) The rotational and pivot frequencies increase with the leg separation $R$, while the translation frequency is independent of that dimension; this effect may be interpreted two ways. First, there is an advantage to increasing $R$ and maximizing $f_{\text {pivot }}$, which is always the lowest frequency. This frequency increases slowly with $R$, however, asymptotically approaching $f_{\text {tran }} / \sqrt{1.5}$. While small changes may often be made to $R$ without adding significant mass to the scanhead, there will be diminishing and even deleterious returns if $R$ is made too large. Second, the designer may choose to focus attention on increasing $f_{\text {rot }}$, so that it is at least equal to $f_{\text {tran }}$ (Fig. 6). Increasing $R$ in this manner will maximize the second-lowest resonant frequency and minimize the noise transmitted by that mode. Again, substantial increases in $R$ may require changes to other dimensions of the scanhead, resulting 


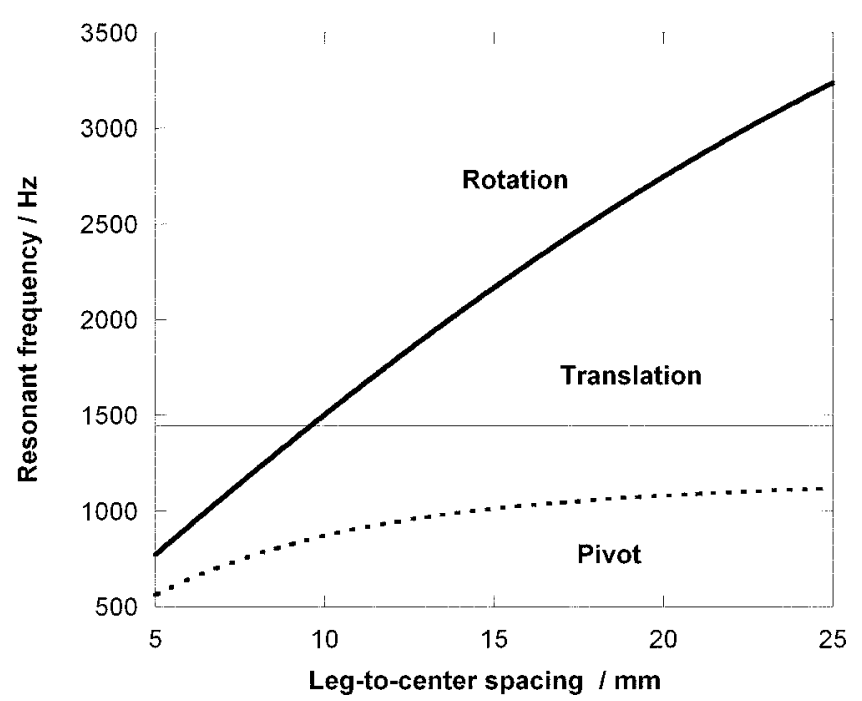

FIG. 6. Increasing the distance from the piezo legs to the center of the disk will increase the natural frequency for the rotation mode but not the translation mode (values are for the scanhead described in the text and Ref. 19).

in additional mass. The implementation of this design rule may therefore be an iterative process.

\section{EXPERIMENTAL TESTING}

The piezoelectric effect method of Behler et al. ${ }^{12}$ (recently reproduced by Pertaya et al. ${ }^{17}$ ) has previously been used to experimentally determine the frequencies of vibration of beetle SPMs. In this method, specific electrodes of the piezo legs are driven by a function generator, with the capacitively induced voltages of the remaining electrodes measured by a lock-in amplifier. This approach proved unsatisfactory for us, as clean and reproducible spectra were not obtained. The summation and amplification of small capacitive signals require careful electronic measurement techniques and multiple rearrangements of connectors to the piezo sectors. Using the laser/cantilever/photodetector system itself as an optomechanical pickup was simple, effective, reproducible and yielded better signal to noise. With an AFM cantilever in place and the optics aligned to produce a standard photodetector signal, a suitable subset of the transverse leg electrodes were again driven by a function generator at low voltage $\left(1 \mathrm{~V}_{\mathrm{PP}}= \pm 9 \mathrm{~nm}\right.$, small compared to the $\pm 130 \mathrm{~V}$ range of the scan control electronics). Normal and lateral force signals were filtered by a lock-in amplifier (SRS 830), with the function generator output used as reference signal. An advantage to this method is the ability to quantitatively determine the amplitude of the cantilever's oscillation, which ultimately is the quantity of interest when considering the impact of microscope vibrations. If the sensitivity of the optical detection system is known (detector response per unit of cantilever deflection) then the power spectral density is easily rescaled to cantilever deflection as a function of excitation frequency. This approach is similar to the technique Jericho et al. ${ }^{18}$ used to characterize an STM, in which one piezo was actuated and the tunneling current was used as a detector.

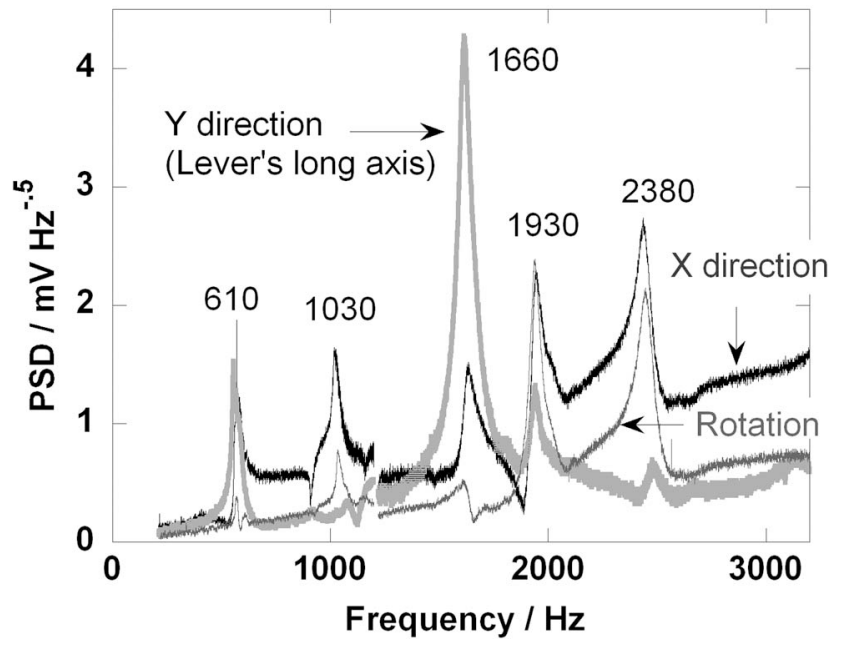

FIG. 7. Power spectral density of scanhead responses to driven oscillation in translation mode (along $X$ and $Y$ axes) and rotation mode, measured by the AFM photodetector's normal force channel.

Using voltage dividers, motion was chosen to be perpendicular to the long axis of the cantilever $(X)$, parallel to that axis $(Y)$, and tangentially about the vertical axis running through the center of the scanhead $(R)$. The out of plane bending of the cantilever [normal force (NF)] was a more effective motion detector than torsional deflection [lateral force (LF)], which is due to the much lower stiffness of the normal force constant of the cantilever. In this method, the vibration of the scanhead at resonance couples to the vibration of the cantilever, allowing sensitive measurements of the net effect of these resonances on the actual motion of the cantilever while out of contact. The lowest resonance frequency of the cantilever itself was on the order of $10 \mathrm{kHz}$ - significantly greater than all driving actuation frequencies. Resonances of the cantilever therefore do not contribute to the measurement.

Frequency sweeps were taken in two stages, 200-1200 and $1200-3200 \mathrm{~Hz}$, each spanning $100 \mathrm{~s}$ and recorded on a digital oscilloscope. Small temporal mismatches of approximately $0.1 \mathrm{~s}$ between initiating the sweeps and the oscilloscope recordings led to gaps of about $10 \mathrm{~Hz}$ in the data at $1200 \mathrm{~Hz}$. This detection method yielded peaks at 610, 1030, 1660, 1930, and $2380 \mathrm{~Hz}$ in all three driving modes (Fig. 7). Experimental uncertainties were $\pm 20 \mathrm{~Hz}$ for each peak position except the last, which was $\pm 40 \mathrm{~Hz}$. Vibration for driving in $Y$ was greatest at $1660 \mathrm{~Hz}$, while driving in $X$ and $R$ both yielded the largest peaks at 1930 and 2380 .

The 21 fine copper wires that connect the control electronics to the piezo legs were not considered in the model, but they do add a small amount of stiffness, increasing the natural frequencies of vibration. They also transmit vibrations from the environment to the scanhead. Other observed modes might be attributed to the low frequency motion of the entire scanhead with respect to the sample stage, which can occur if the contact between the walker and the stage is small, due to having a weak mechanical contact. This cannot be known a priori, but this effect was used to explain several intermittent low frequency vibrations by Behler et al., ${ }^{12}$ who observed this for their particularly small, light beetle scan- 
ning tunneling microscope (STM) head. Finally, resonances of small, individual mechanical components on our scanhead, including the spring-loaded photodiode holder, the fiber optic mounting piece, and the cantilever stem and holder, could also produce these peaks.

\section{DISCUSSION}

The predicted resonant frequencies of translation, rotation, and pivoting for our scanhead are 1450, 1920, and $970 \mathrm{~Hz}$, respectively. Because the scanhead was already assembled and connected to delicate electronic leads, the masses of the individual components could not be determined independently, so rough values of $M$ and $J$ were calculated from the geometry. ${ }^{19}$ Consequently, there is appreciable uncertainty in the masses and moments of the more irregularly shaped components. Manufacturer values of $E$ and $\rho$ for PZT were used in the calculations.

Motion in $Y$ produces by far the largest peak at $1660 \mathrm{~Hz}$. This mode is parallel to the lever's long axis and would couple most efficiently to the lever's flexural vibration, showing up most strongly in the measured normal force channel. We therefore conclude that the translation mode resonates at $1660 \mathrm{~Hz}$. The prediction of a peak at $1450 \mathrm{~Hz}$ is thus an underestimation by $14 \%$. The response to driving in $R$ is greatest at $1930 \mathrm{~Hz}$, in remarkable (and perhaps somewhat fortuitous) agreement with the predicted resonance at $1920 \mathrm{~Hz}$. Last, the peaks in $Y$ and $R$ at $1030 \mathrm{~Hz}$ are in very good agreement with the predicted pivot peak at $970 \mathrm{~Hz}$.

One interesting feature of the $1030 \mathrm{~Hz}$ pivot peak is that cantilever's deflection is greater in response to $X$ excitation than $Y$ excitation, despite the orientation of the cantilever, a result of the threefold symmetry of the scanhead. Translation in the $X$ direction is very similar to a pivot motion about the front leg (as shown in Fig. 3), so strong coupling of the drive to the cantilever motion is expected. Translation along the $Y$ direction could couple to a pivot action about either of the two rear legs, but the plane of symmetry running through the front leg results in those two pivot motions canceling each other out and reducing the motion of the scanhead. Therefore, the decreasing strength of the response at the pivot mode frequency to drive in $R, X$, and $Y$ follows what we would predict based on the geometry, bolstering the conclusion that this frequency represents the pivot mode.

The smaller responses at 610 and $2380 \mathrm{~Hz}$ are of unknown origin, but the fact that the latter is not excited when driving the head along the $Y$ axis suggests that it is related to rotation or it may be related to a harmonic of the pivot mode (which would theoretically be at about $2900 \mathrm{~Hz}$ ). There are additional degrees of freedom in the scanhead not considered in this analysis. In particular, contacts holding the photodetector and cantilever in place are of finite and unknown stiffness. Also, the components of the scanhead, such as the lens and the photodetector, are not rigid and may vibrate independently of the piezo legs. The peak at $600 \mathrm{~Hz}$ was witnessed by both the piezo pickups and the photodetector and at two stages in the scanhead's construction (in its final state and before the detector, cantilever holder, and central stem were added). We believe it is not an artifact of the measuring process and that its excitation frequency does not depend on the mass of the scanhead.

An additional limitation to this analysis is that the central disk and probe equipment are assumed to be axially symmetric, but as is evident in Fig. 1, that is not the case. Thus, the center of mass of the disk does not actually lie on the central axis of the scanhead, a factor which will contribute to coupling between translational and rotational vibration modes. The alternate piezo pickup scheme of Behler et al. is flexible in that selecting the proper polarity of the twelve available pickup electrodes allows motion in a specific direction (or around a particular axis) to be monitored, but our optical method lacks the required degrees of freedom to accomplish that. Therefore we cannot say with complete certainty that $1930 \mathrm{~Hz}$ represents rotation and $1660 \mathrm{~Hz}$ represents translation, but the agreement with the predicted values lends credence to this claim. Also, we expect the cantilever to respond more strongly to drive in the $Y$ direction as compared with $X$ because its axis lies along $Y$. Indeed, the response at $1660 \mathrm{~Hz}$ is three times larger for $Y$ vs $X$ drive, strengthening the claim that $1660 \mathrm{~Hz}$ represents the translation mode. The advantage of our detection scheme is that it makes use of the built-in displacement sensing of the AFM itself and no additional electronic components or modifications to the electronic connections are needed to carry out the measurements.

The design of an SPM necessarily involves trade-offs: rigidity comes at the cost of some added bulk, longer legs mean larger scans but reduced stability, and additional features require more mass. Eigenfrequency analysis and design rules are presented here with the goal of assisting microscope builders in making their choices so that designs may be optimized. Our general frequency predictions for two previously discussed and one new mode of vibration help to accurately circumscribe the design space. They include the nontrivial inertial terms of the piezo actuators' mass and the precise deformation modes and boundary conditions of the actuators. The predicted modes of vibration are in close agreement with those witnessed experimentally.

\section{ACKNOWLEDGMENTS}

The authors would like to thank R. J. Cannara for experimental assistance, M. E. Plesha and D. C. Kammer for helpful discussions, and the National Science Foundation (CAREER Award No. CMS-0134571 and Graduate Student Research Fellowship) for support of this work.

\section{APPENDIX}

As scanheads are made lighter, the effects of leg mass on the vibration frequencies will become more pronounced. Here, we demonstrate an $18 \%$ effect by using a lighter, stiffer material and by rescaling thicknesses of disk assembly components to take advantage of the material's stiffness. The changes made are as follows:

(1) Switch the material to beryllium (which is significantly lighter and stiffer than $\mathrm{Al}$ ). Although beryllium has 
known serious health effects, it is indeed used for high performance mechanical applications and is not an unrealistic material to consider.

(2) Use the reduced mass and increased stiffness of Be to make the disk thinner to the point that the "drumhead" vibration mode of the circular disk is equivalent to that of the original $\mathrm{Al}$ scanhead. In fact, this could be further reduced to bring the vibration frequency of the drumhead mode even lower but still above the lowest resonance frequencies of the head. For simplicity we forgo this analysis here.

(3) The figure of merit for the frequency of a circular plate $\mathrm{e}^{13}$ is $E h^{2} /\left[12 \rho\left(1-\nu^{2}\right)\right]$ where $E=$ Young's modulus, $\rho$ $=$ density, $h=$ thickness, and $\nu=$ Poisson ratio. Given the differences in $E, \nu$, and $\rho$ for the two materials, the new thickness that maintains the same resonance frequency is $h_{\mathrm{Be}}=0.43 h_{\mathrm{Al}}$.

(4) The total mass of the disk becomes $M_{\text {disk.Be }}$ $=M_{\text {disk,Al }} 0.43\left(\rho_{\mathrm{Be}} / \rho_{\mathrm{Al}}\right)=0.3 M_{\mathrm{Al}}$. We may then find the rotation, translation, and pivot mode frequencies using (8) and (9) and compare with the formulas from Behler et al., resulting in $18 \%$ correction for rotation and $10 \%$ for translation for the Be scanhead.

${ }^{1}$ G. Meyer, Rev. Sci. Instrum. 67, 2960 (1996).
${ }^{2}$ RHK Technology, Troy, MI; SPS-Createc, Erligheim, Germany; Nanotec Electrónica, Madrid, Spain.

${ }^{3}$ C. J. Chen, Appl. Phys. Lett. 60, 132 (1992).

${ }^{4}$ J. Frohn, J. F. Wolf, K. Besocke, and M. Teske, Rev. Sci. Instrum. 60, 1200 (1989).

${ }^{5}$ D. W. Pohl, Rev. Sci. Instrum. 58, 54 (1987).

${ }^{6}$ Q. Dai, R. Vollmer, R. W. Carpick, D. F. Ogletree, and M. Salmeron, Rev. Sci. Instrum. 66, 5266 (1995).

${ }^{7}$ D. W. Pohl, IBM J. Res. Dev. 30, 417 (1986).

${ }^{8}$ S.-I. Park and R. C. Barrett, J. Mater. Eng. Perform. 27, 31 (1993).

${ }^{9}$ F. P. Beer and E. R. Johnston, Jr., Mechanics of Materials, 2nd ed. (McGraw-Hill, New York, 1992).

${ }^{10}$ L. Meirovitch, Fundamentals of Vibrations (McGraw-Hill Higher Education, New York, 2001).

${ }^{11}$ W. T. Thomson, Theory of Vibration with Applications, 4th ed. (PrenticeHall, Upper Saddle River, NJ, 1993).

${ }^{12}$ S. Behler, M. K. Rose, D. F. Ogletree, and M. Salmeron, Rev. Sci Instrum. 68, 124 (1997).

${ }^{13}$ R. D. Blevins, Formulas for Natural Frequency and Mode Shape (Krieger, Malabar, FL, 1995).

${ }^{14}$ K. L. Johnson, Contact Mechanics (Cambridge University Press, Cambridge, New York City, 1985).

${ }^{15}$ J. MacLeod, A. Moffat, J. Miwa, A. Mark, G. Mullins, R. Dumont, G. Constant, and A. McLean, Rev. Sci. Instrum. 74, 2429 (2003).

${ }^{16}$ J. A. Miwa, J. M. MacLeod, A. Moffat, and A. B. McLean, Ultramicroscopy 98, 43 (2003).

${ }^{17}$ N. Pertaya, K. F. Braun, and K. H. Rieder, Rev. Sci. Instrum. 75, 2608 (2004).

${ }^{18}$ M. H. Jericho, D. C. Dahn, and B. L. Blackford, Rev. Sci. Instrum. 58, 1349 (1987).

${ }^{19} M=10.3 \mathrm{~g}, J=948 \mathrm{~g} \mathrm{~mm}^{2}, R=13 \mathrm{~mm}, L=13 \mathrm{~mm}, A_{x s}=4.7 \mathrm{~mm}^{2}$. 